
\title{
$\begin{array}{ll}\text { Research Square } & \begin{array}{l}\text { Preprints are preliminary reports that have not undergone peer review. } \\ \text { They should not be considered conclusive, used to inform clinical practice, } \\ \text { or referenced by the media as validated information. }\end{array}\end{array}$
}

\section{Quantitative Proteomics Identifies Cannabinoid Receptor 2 as Candidate Marker of Idiopathic Pulmonary Fibrosis and Dysregulation of Endogenous Cannabinoids Pathway}

\section{wenjing du}

Huashan Hospital Fudan University Institute of Integrative Medicine: Huashan Hospital Fudan University Department of Integrative Medicine

\section{Ting Zhang}

Huashan Hospital Fudan University Institute of Integrative Medicine: Huashan Hospital Fudan University Department of Integrative Medicine

\section{Fangyong Yang}

Huashan Hospital Fudan University Institute of Integrative Medicine: Huashan Hospital Fudan University Department of Integrative Medicine

\section{Zhao Tang}

Huashan Hospital Fudan University Institute of Integrative Medicine: Huashan Hospital Fudan University Department of Integrative Medicine

\section{Qiuping Li}

Huashan Hospital Fudan University Institute of Integrative Medicine: Huashan Hospital Fudan University Department of Integrative Medicine

\section{Aman Gul}

Huashan Hospital Fudan University Institute of Integrative Medicine: Huashan Hospital Fudan University Department of Integrative Medicine

\section{Hu Zhang}

Huashan Hospital Fudan University Institute of Integrative Medicine: Huashan Hospital Fudan University Department of Integrative Medicine

\section{La Yi}

Huashan Hospital Fudan University Institute of Integrative Medicine: Huashan Hospital Fudan University Department of Integrative Medicine

\section{Shiqiang Wang}

Shanghai PuTuo District Center Hospital

Jingcheng Dong ( $\nabla$ jcdong2004@126.com )

Fudan University 
Research

Keywords: Idiopathic pulmonary fibrosis, Cannabinoid receptor 2, metabolomics, Icariin

Posted Date: September 22nd, 2021

DOl: https://doi.org/10.21203/rs.3.rs-900435/v1

License: (c) (i) This work is licensed under a Creative Commons Attribution 4.0 International License. Read Full License 


\section{Abstract}

Background: Idiopathic pulmonary fibrosis (IPF) a chronic, progressive, and lung fibrosis disease of unknown etiology with less effective treatment. It is important to discover new biomarker and therapeutic target for the diagnosis and cure of IPF.

Method: Differential metabolic profiles may be useful for the diagnosis of IPF and provide additional insight into the molecular mechanisms underlying IPF. Plasma samples from IPF, COPD and normal controls were investigated using liquid chromatography-quadrupole time-of-flight mass spectrometry (UHPLC/Q-TOF-MS) and these datasets were analyzed using multiple pattern performance methods. Multivariate statistical methods, pathway enrichment analysis and univariate receiver operating characteristic (ROC) curve analysis were performed.

Results: OPLS-DA results showed that it exhibited significant separation between any two groups. ROC curve analyses revealed that 8 metabolites with high AUC above 0.7 between three groups in the plasma samples. Pathway analysis revealed that 3 metabolites are involved in endogenous cannabinoids/cannabinoid receptor 2 (CB2) signaling. Moreover, we found that the specific elevation of CB2 could be a signature of PF(Pulmonary Fibrosis) lung tissues in rat model.

Conclusions: LC-MS-based plasma metabolomics provides a important tool to identify the potential biomarkers for IPF. Taken together, we performed quantitative chemoproteomic profiling and identified endogenous cannabinoids/CB2 as the key target of ICA in bleomycin-induced pulmonary fibrosis.

Trial Registration: The study was approved by the the Ethics Committee of Huashan Hospital, Fudan University (KY2021-453)

\section{Background}

Idiopathic pulmonary fibrosis (IPF) is a progressive fibrotic lung disease characterized by excessive proliferation of fibroblasts and extracellular matrix accumulation that leads to the distortion of the lung parenchyma and the loss of lung function[1,2]. With a median survival of 3-5 years following diagnosis, IPF Patients usually present unspecific symptoms, such as cough and dyspnea on exertion. The diagnosis of IPF is based on typical features of high-resolution computed tomography (HRCT) or lung biopsy excluding other idiopathic interstitial lung diseases (ILD) in a multidisciplinary setting[3, 4]. The lung biopsy is invasive and has several risk procedures in those patients with an uncertain diagnosis and those thought to have IPF[5, 6]. Moreover, IPF is a chronic and fetal interstitial lung disease with poor prognosis and limited treatment options $[3,7,8]$. Pirfenidone and Nintedanib are two anti-fibrotic therapies available for the treatment of IPF and have been approved since 2014[9, 10]. Hence, exploring the molecular mechanisms involved in the development of pulmonary fibrosis may lead to novel therapeutic strategy. 
Metabolomics is the study of inputs and outputs of biological pathways and often considered important representative of the function state of a cell or organ. Currently, an untargeted metabolomics analysis can detect many metabolites at the same time. To identify novel pathway or diagnosis biomarkers indicative of metabolic alterations, metabolomics is a cumulatively used experimental method. In this study, we try to identify the potential plasma metabolic biomarker used for diagnosing IPF.

\section{Materials And Methods}

\section{Human subjects}

All human plasma samples of patients with non-acute exacerbation IPF $(n=10)$, stable chronic obstructive pulmonary disease (COPD) $(n=10)$, and normal control $(N C)$ subjects $(n=10)$ were obtained from Huashan Hospital, Fudan University and stored by $-80^{\circ} \mathrm{C}$ refrigerator until analysis. IPF was diagnosed according to the international guideline 2011 and 2018[3, 4]. COPD was diagnosed according to the the Global Initiative for Chronic Obstructive Lung disease[11]. The normal subjects had no appearance or history of respiratory diseases and malignant tumor. Data from the clinical history and physical examination were analyzed, as well as the results of the lung function, blood gas determination (Table 1). The study protocol was approved by the Ethics Committee of Huashan Hospital, Fudan University (Shanghai, China). 
Table 1

Baseline Characteristics of Study Subjects

\begin{tabular}{|c|c|c|c|}
\hline & IPF $(n=10)$ & $\operatorname{COPD}(n=10)$ & $N C(n=10)$ \\
\hline Age years & $61.5 \pm 11.63$ & $65 \pm 7.26$ & $56.5 \pm 5.19$ \\
\hline \multicolumn{4}{|l|}{$\operatorname{Sex}(\square)$} \\
\hline Female & 3 & 2 & 3 \\
\hline Male & 7 & 8 & 7 \\
\hline \multicolumn{4}{|l|}{ Complicating disease } \\
\hline Hypertension (n) & 1 & 3 & 0 \\
\hline Type 2 Diabetes (n) & 1 & 2 & 0 \\
\hline Smokers (n) & 3 & 8 & 2 \\
\hline \multicolumn{4}{|l|}{ Lung function } \\
\hline FVC (\% predicted) & $79.49 \pm 14.87$ & $63.77 \pm 16.71$ & - \\
\hline FEV1 (\% predicted) & $78.78 \pm 11.05$ & $41.76 \pm 9.33$ & - \\
\hline FEV1/FVC (\% predicted) & $99.29 \pm 10.13$ & $59.53 \pm 8.43$ & - \\
\hline \multicolumn{4}{|l|}{ Arterial blood gas analysis } \\
\hline $\mathrm{SpO}_{2}(\%)$ & $92.36 \pm 5.42$ & $97.07 \pm 1.4$ & - \\
\hline $\mathrm{pO}_{2}(\mathrm{kPa})$ & $9.16 \pm 2.93$ & $14.84 \pm 3.12$ & - \\
\hline $\mathrm{pCO}_{2}(\mathrm{kPa})$ & $5.31 \pm 0.19$ & $6.87 \pm 1.2$ & - \\
\hline
\end{tabular}

\section{Sample preparation}

Frozen plasma was thawed at $4^{\circ} \mathrm{C} .400 \mu \mathrm{l}$ methanol-acetonitrile $(1: 1, \mathrm{v} / \mathrm{v})$ solution was added to $100 \mu \mathrm{l}$ plasma in a $1.5 \mathrm{~mL}$ microcentrifuge tube. Samples were vortexed for $60 \mathrm{~s}$, stored at $-20^{\circ} \mathrm{C}$ for 1 hour to precipitate protein, and then centrifugation for $20 \mathrm{~min}$ at $12000 \mathrm{rpm}$ at $4^{\circ} \mathrm{C}$. Subsequently, the supernatant was isolated and lyophilized. Methanol solution was added to reconstitute the dried residue. Following centrifugation for $15 \mathrm{~min}$ at $12000 \mathrm{rpm}$ at $4{ }^{\circ} \mathrm{C}$, isolation of the supernatant for LC-MS analysis. A pool quality control (QC) sample was aliquoted from the pooled group samples.

\section{UPLC-Q-TOF-MS analysis}

The experimental samples were analyzed by an Agilent 1290 UPLC system (Agilent Technologies, CA, USA) coupled to Triple TOF 5600 (AB SCIEX, CA, USA). ACQUITY UPLC BEH Amide Column $(2.1 \mathrm{~mm} \times 100$ 
$\mathrm{mm}, 1.7 \mu \mathrm{m}$, Waters, USA) at a flow rate of $0.3 \mathrm{~mL} / \mathrm{min}$. The mobile phase was composed of ammonium acetate $(A)$ and acetonitrile (B). The following LC gradient was applied: 0-0.5 min: 95\% B, 0.5-7 min: 95\%-65\% B, 7-8 min: 65\%-40\% B, 8-9 min: 40\% B, 9-9.1 min: 40\%-95\%, 9.1-12 min: 95\% B. MS analysis was conducted with a Triple TOF 5600 system with electrospray ionization (ESI) operating in positive (ESII) and negative (ESI-) modes. Ion sapary voltages floating (IAVF): $\pm 5500 \mathrm{~V}$, TOF MS scan $\mathrm{m} / \mathrm{z}$ range: 60-1000 Da. The entire sample loading process is carried out by the autosampler at $4^{\circ} \mathrm{C}$, with QC samples inserted at regular intervals.

\section{Raw Data analysis, metabolite identification and pathway analysis}

The raw data files were converted into the $\mathrm{mzXML}$ format, and then using the XCMS package to perform peak alignment, retention time correction and extraction peak area[12]. Prior to multivariate statistical analysis, metabolite data were subjected to pareto-scaling. Principal component analysis (PCA) and orthogonal partial least squares discriminant analysis (OPLS-DA) were performed for pattern recognition analysis. In the OPLS-DA model, the variable importance in projection (VIP) score were calculated. The student's t-test was selected for pairwise comparison. The significantly changed features were screened with VIP $₫ 1$, and $P$ value $₫ 0.05$ was considered to be statistically significant. Significantly changed features and pathway analysis were compared with the built-in database by searching KEGG (http://www.genome.jp/kegg/)[13].

\section{Reagents and antibodies}

Acetonitrile and methanol were acquired from Merck (Frankfurt, Germany). Bleomycin (BLM) was purchased from Nippon Kayaku Co. Ltd (Tokyo, Japan). The experimental cigarettes were purchased from Kentucky Tobacco Research \& Development Center (Kentucky, USA). Anti-Cannabinoid Receptor 2 (ab3561) antibody was purchased from Abcam (Cambridge, MA, USA), Cannabinoid receptor 1 (17978-1AP) was purchased from Proteintech Group. Inc (Rosemont, IL, USA).

\section{Animals and treatment}

All animal experimental protocols were approved by the Animal Care and Use Committee of Fudan University (Shanghai, China). Male SD rat with mean weight 200-220g were obtained from Shanghai SLAC Laboratory Animal Centre (Shanghai, China), and then adaptive feeding for three days. 20 rats were randomly allocated to control group and BLM group $(n=10$ per group). Rat pulmonary fibrosis model was induced by BLM. BLM was administered once by intra -tracheal instillation at $3 \mathrm{U} / \mathrm{kg}$ in $0.9 \%$ saline. At the end of the study, the rats were sacrificed by pentobarbitone overdose, and then lung tissues were collected. The construct protocol of Chronic obstructive pulmonary disease (COPD) rat modle was described in the our previous article[14].

\section{In vivo micro-CT analysis}

In vivo micro-CT examinations of rat lungs were carried out at the end of the study. CT images were acquired in high resolution mode on the trimodal Quantum GX micro-CT scanner (PerkinElmer, Waltham, 
MA., USA) using the parameters as follow: Voltage: 89 kV, Current: $86 \mu \mathrm{A}$, Dose: 219 mGy, CT: 88, FOV: 72 $\mathrm{mm}$, Voxel Size: $144 \mu \mathrm{m}$, Scan Time: 4 min. Built-in software was used to reconstruct the resulting CT images.

\section{Lung histology}

Lung tissues were fixed in paraformaldehyde solution for $48 \mathrm{~h}$, embedded in paraffin, and then sectioned at $8 \mu \mathrm{m}$ thickness. The lung slides were stained with hematoxylin-eosin (H\&E) and Masson's trichrome. After dewaxed, heated in the antigen retrieval reagent and blocked with goat serum, the lung sections were incubated with primary antibody CB 2 (1:50, ab3561, Abcam, Cambridge, MA, USA), CB 1 (1:400, 17978-1-AP, Proteintech, Rosemont, IL, USA) and a-SMA (1:600, \#19245, CST, Beverly, MA, USA) overnight at $4{ }^{\circ} \mathrm{C}$. The next day, sections were incubated with HRP-conjugated secondary antibodies or fluorescence-conjugated secondary antibody. The nuclear staining with DAPI (C1005, Beyotime, Shanghai, China) for $10 \mathrm{~min}$. The stained sections were scanned with S360 NanoZoomer Digital slide scanner (Hamamatsu, Shizuoka Pref, Japan), and pictures were taken with NDP.view2 software. The expression of Anti-Cannabinoid Receptor 2 (CB2) and Anti-Cannabinoid Receptor 1 (CB1) were investigated by Immunohistochemical $(\mathrm{IHC})$ analysis,and the expression of a-SMA was determined by Immunofluorescent (IF) analysis.

\section{Results}

\section{Characteristics of subjects and plasma metabolic profiling by UPLC-Q-TOF-MS}

Characteristics of the study population (patients with IPF, COPD and normal control) were presented in Table 1. To search the metabolic differences among IPF, COPD and normal control, we applied nontargeted LC-MS focusing on the metabolic profiles of the plasma samples and the related specific metabolism pathways underlying IPF development. In order to identify the real biological differences, it is necessary to perform effective qualitative control (QC). The overlapping spectra was conducted for the UPLC-Q-TOF-MS total ion chromtograms of QC samples (Fig. 1A). Scatter plots and regression lines demonstrated correlation of ion current intensity of QC samples (Fig. 1B). All in all, these data showed that the retention time and ion current intensity of chromatographic peaks overlapped, indicating that the differences found in this study were biological differences rather than technical differences caused by measurement errors. Moreover, PCA analysis of the metabolic profiles measured by UPLC-Q-TOF-MS indicated differences between IPF patients, COPD patients and healthy control group (Fig. 1C). Hotelling's T2 analysis was employed to determine whether an outlier sample exists. It showed that most samples were within the $99 \%$ confidence interval (Fig. 1D). Taken together, results show that the graphical integration of outputs from multiple quantitative tools facilitates the evaluation of instrumental effects and judges the necessity for further data analysis.

Metabolomics analysis of plasma samples from normal control, IPF and COPD subjects. 
PCA and OPLS-DA were performed to determine whether it was possible to distinguish NC, IPF and COPD subjects on the basis of the metabolic data. Obviously, PCA analysis showed that there was no significant separation among these three groups (Figure. 2A). However, OPLS-DA results showed that it exhibited significant separation between any two groups and then the replacement inspection established a 200 -fold OPLS-DA model by randomly changing the order of classification variable $Y$ to obtain the R2 and Q2 values of a random model (Fig. 2B: left panel). All of these blue Q2 points, which were shown in left to right, were lower than the original Q2 points in positive ion mode (Fig. 2B: right panel). The same results were obtained in negative ion mode (Fig. $2 \mathrm{C}$ ). All in all, these results suggested that the model was reliable without fitting.

\section{Selection of potential metabolite markers for IPF}

Box plots and classical univariate ROC curve analyses were used to further characterize the predictive value of these individual metabolites independently. The box plots showed the relative changes in the eight potential metabolites D-Proline, Deoxycholic acid, EDTA, Erucamide, Glycochenodeoxycholate, Myristic acid, Taurochenodeoxycholate and 11(Z),14(Z)-Eicosadienoic Acid between groups (Fig. 3A). Furthermore, ROC curves also showed a apparent discrimination of these eight metabolites between Groups with an area under the curve (AUC) $>0.7$, as shown in Fig. 3B and Fig. 3C. The results of ROC curve analyses suggested that 8 metabolites with high AUC above 0.7 between groups in the plasma samples.

\section{Screening and identification of differential metabolites}

The metabolite features were selected as VIP $>1.0$ and $P$ value $<0.1$. As shown in Volcano plot Fig. 4A, the differential metabolities significantly up or down-regulated among NC, IPF and COPD (FC $>1.5$ and P value $<0.05$ ). The heatmap and hierarchical cluster analysis showed the distribution of significant differential metabolites between groups in the IPF, COPD and NC (Figure. 4B). Box plots showed representative metabolite changes among NC, IPF and COPD groups (Fig. 4C). Compared with the IPF and COPD group, the NC group had higher levels of 3 -Indoleacetonitrile ( $p<0.05$; ${ }^{*} p<0.01$ ).

Glycochenodeoxycholate level was higher in IPF samples. However, Vanillin was significantly lower in NC samples. In order to understand the relationship of metabolite differences with IPF, correlation analysis was used to analyze the differential metabolites (Fig. 4D). These data showed that the individual discriminating metabolite may be useful to differentiated the IPF from NC and COPD patients, and the accuracy can be further up-regulated if more pre-selected discriminating metabolites would be used.

\section{Pathway enrichment analysis and metabolite signatures reflecting aberrant metabolism in IPF}

The KEGG pathway enrich analysis of the differential metabolites between the IPF, COPD, and NC groups were identified. The top 20 most enriched pathway terms are shown in the KEGG enrichment bubble diagrams (Figure. 5A). The main differential pathways in IPF compared to other groups indicated that the differential metabolites were mainly involved in the following pathways: Central carbon metabolism in cancer, Protein digestion and absorption, Aminoacyl-tRNA biosynthesis, Retrograde endocannabinoid 
signaling, and Linoleic acid metabolism, et al. The key differential metabolites between IPF and NC were identified to be involved in the pathways for Retrograde endocannabinoid signaling (Fig. 5B). Moreover, Box plots showed an apparent discrimination of the three metabolites associated with retrograde endocannabinoid signaling among the three groups (Fig. 5C).

Previous studies have shown that human lung-resident macrophages expressed a complete endocannabinoid system, the cannabinoid receptors was also expressed in lung cancer-associated macrophages and cannabinoid receptor activation selectively inhibited the release of angiogenic and chronic inflammation[15]. Recent studies shown that CB1 expression by ATIl cells and alveolar macrophages was indicated by their colocalization with surfactant protein C (SP-C) and CD68 respectively[16]. However, little is known about cell-type-specific localization and the function of CB2 in BLM-induced lung fibrosis. To further examine the relationship between the endocannabinoid signaling and pulmonary fibrosis, we detected the $\mathrm{CB} 2$ receptor protein expression in the lung tissues of rat models (BLM-induced pulmonary fibrosis, cigarette smoke-induced COPD, and normal control) corresponding to the three groups of clinical subjects. Immunohistochemistry results show that the protein expression of CB2 receptor in the lung tissues of BLM-induced rat pulmonary fibrosis model was significantly upregulated, whereas there was no significant change in the lung tissues of cigarette smoke-induced rat COPD model and normal control rats. Positive immunostaining for CB2 was observed in alveolar epithelial cells and myofibroblasts in lung fibrogenesis (Fig. 5D). Collectively, these results demonstrated that endocannabinoid signaling may associated with pulmonary fibrosis.

\section{Validation of dysregulated endocannabinoid signaling in rate model}

The above results revealed that the endocannabinoid signaling may be associated with pulmonary fibrosis. To validate the findings from the proteomics study, endocannabinoid/cannabinoid receptor system was selected for verification in the lung tissue samples by immunohistochemistry. Next, Highresolution micro-CT scan was carried out to assess lung morphologic changes. The reconstruction of micro-CT image analysis of BLM-induced pulmonary fibrosis rats showed remarkable lung structure destruction as compared to normal control rats, as illustrated in Fig. 6A. BLM instillation resulted in histological damage of lungs tissues in BLM-induced pulmonary fibrosis rats, characterized by consolidation of the parenchyma and the excessive deposition of collagen. a-SMA immunostaining of lung tissue was significantly increased in PF group, relative to Normal control (NC) group. In contrast, BLM-induced a-SMA expression was attenuated by the treatment of ICA (Fig. 6B).

In order to determine the role of $\mathrm{CB} 2$ receptor activation mediating the effect of BLM-induced lung fibrosis. As shown in Fig. 6C, we examined the CB1 and CB2 protein expression by using immunohistochemical staining. The expression of $\mathrm{CB} 2$ receptor protein was significantly up-regulated in the PF group after BLM instillation. Moreover, the expression of $C B 2$ protein was predominantly in alveolar epithelial cells and myofibroblasts. Taken together, the BLM-induced increase in CB2 levels was dramatically changed, and reflected the key role of CB2 blockade in treatment of IPF. 


\section{Discussion}

The pathogenesis of IPF involved a series of pathological process, such as early inflammation, oxidative stress, myofibroblasts proliferation, excessive wound healing and fibrosis. Deficient understanding of the mechanisms underlying IPF has hampered the development of efficient tools for diagnosis and interventions[17]. For the discovery of new biomarkers, the application of metabolomics is emerging and significantly contributes to a deeper understanding of the metabolic pathways in IPF [18]. Moreover, previous studies have showed that dysregulated metabolism may be used to discover new biomarkers and targets[19]. In this work, a simple, repeatable and sensitive method was validated for the simultaneous determination of metabolites for diagnosis of IPF disease. Herein, a non-targeted UPLC-QTOF-MS plasma metabolism method was applied for exploring the metabolic characteristics to screen efficient predictors of IPF. The presented results demonstrated the metabolic profiling of IPF patients differing from those of controls and COPD, which showed satisfying data quality. We compared plasma metabolic profiles of 30 subjects to identify its metabolic signatures. Previous studies on the metabolites of the three groups have been really rare.

Previous study reported that the advantage of plasma metabolic profiling for characterizing metabolite signature of IPF and changes in metabolic pathways that might be helpful for understanding the metabolic mechanism of pulmonary fibrosis[20]. In the current study, PCA did not show a clearly different distribution in the positive/negative ion model. Then, we applied OPLS-DA statistical approach to select differential metabolites. 47 significantly changed metabolites related to IPF were found to be differentialy altered among the three groups in positive/negative ion model. Furthermore, recent many studies have been proved that establishing a diagnostic model to predict the IPF patients could potentially improve the diagnosis[21]. ROC curves of classification models base on the 8 metabolites with high AUC above 0.7 were plotted to distinguish IPF and normal control. Therefore, a combination of more than one discriminatory metabolite will be necessary to increase the diagnostic performance of IPF. To research their metabolism mechanisms, the enriched pathways of the metabolites were also analyzed according to the KEGG database, which reflected the key pathway related with differential metabolites. The most enriched pathway terms were biosynthesis of unsaturated fatty acids, caffeine metabolism, Arginine biosynthesis, linoleic acid metabolism, central carbon metabolism in cancer, protein digestion and absorption, retrograte endocannabinoid signaling and $A B C$ transporters.

Endocannabinoid, interacts with CB1 and CB2 receptors of endocannabinoid system, help coordinate and regulate everything which we feel, think and do. The endocannabinoid/cannabinoid receptor system may be a rational therapeutic target in IPF and promote inflammatory by CB1 activation in many chronic inflammatory diseases[20]. In addition to promoting inflammatory, activation of CB1 also promote fibrosis progression in many organs, such as liver, kidney, heart and skin[22-24]. Simultaneously, it is reported that the brain-penetrant CB1 antagonist attenuates liver fibrosis in mice model[25]. However, the potential role of $\mathrm{CB} 2$ has not yet been investigated in patients with IPF or pulmonary fibrosis animal model. As shown in Fig. 5D, CB2 is predominantly expressed in ATIl and myofibroblast cells of lung. Rice W et al. reported that CB1 is always expressed in ATII cells, bronchial epithelial cells, and alveolar 
macrophages[26]. However, we found a higher expression of CB1 in alveolar macrophages than other cell populations following BLM treatment.

Although the FDA have approved pirfenidone and nintedanib for the cure of IPF patients, both compounds still have modest efficacy, but not lead to good overall survival.Therefore, the ideal application implies that simultaneously targeting alveolar inflammation and fibrotic process.

Polypharmacology may offer a model for the way to drug discovery. As was demonstrated in Fig. 6, it was observed that the CB1 and CB2 were specifically activated in rat PF model. Furthermore, Resat Cinar etal. reported that elevated activity of the endocannabinoid/CB1 system parallels disease progression in the patients with IPF and in mice with BLM-induced lung fibrosis, thus making it a feasible candidate for the treatment of IPF. Next, cannabinoid receptors antagonist or CB2 deletion will be employed to explore the detail mechanism of antifibrosis and find the downstream target of endocannabinoid/cannabinoid receptor system in the lung with PF. In summary, the results of this study indicate that the endocannabinoid/cannabinoid receptor system and related signal transduction molecules are important targets for the diagnosis and treatment of PF, and that the treatment of ICA protect against pulmonary fibrosis induced by BLM in rats for the first time. Further research should be encouraged to clarify the mechanism of endocannabinoid/cannabinoid receptor system and evaluate the effectiveness of ICA in patients with IPF.

\section{Conclusions}

The present study demonstrates that metabolic profiling of plasma by untargeted LC-MS analysis allows to discriminate among NC, IPF and COPD subjects. Therefore, the differential metabolites and enriched signaling pathways may involve in metabolisms of endocannabinoid/cannabinoid receptor system in pulmonary fibrosis. The outcome may be supporting CB2 as potential treatment target for PF.

\section{Abbreviations}

Cannabinoid receptor 2, CB2; Idiopathic pulmonary fibrosis, IPF;bleomycin, BLM; liquid chromatographyquadrupole time-of-flight mass spectrometry, UHPLC/Q-TOF-MS; receiver operating characteristic, ROC; chronic obstructive pulmonary disease, COPD; pulmonary fibrosis, PF; idiopathic interstitial lung diseases, ILD.

\section{Declarations}

\section{Acknowledgements}

UHPLC/Q-TOF-MS analysis was performed at the Shanghai Applied Protein Technology Co., Ltd. (APTBIO). Thanks for all the volunteers and patients; Siqiang for sample collection.

\section{Funding}


This research was funded by the grant from National Natural Science Foundation of China (81600309), Natural Science Foundation of Shanghai (16ZR1404800), The Scientific Research Starting Foundation of North Huashan Hospital Affiliated to Fudan University (2015108).

\section{Availability of data and materials}

Please contact author for data requests.

\section{Author's contributions}

Wenjing Du: Investigation, Data curation. Zhao Tang: Writing - original draft. Fangyong Yang: Investigation. Ting Zhang: Data curation. Xijun Liu: Funding acquisition, data analysis, Writingreview\&editing. Qiuping Li:Investigation. Aman Gul: Investigation. Hu Zhang: Investigation, Data curation. La Yi: Investigation, Shiqiang Wang: collect clinic samples, Jingcheng Dong: Funding acquisition, Conceptualization, Data curation, Supervision.

\section{Ethics approval and consent to participate}

Approval form the local ethical committee has been obtained (Ethics Committee of Huashan Hospital, Fudan University, approval KY2021-453). The plasma samples from IPF and COPD patients has been obtained in Huashan Hospital previous to data acquisition.

\section{Consent for publication}

Not applicable

\section{Competing interests}

The authors declare no conflict of interest.

\section{References}

1. Lederer DJ, Martinez FJ. Idiopathic Pulmonary Fibrosis. New Engl J Med. 2018; 378: 1811-23.

2. Maher TM, Wells AU, Laurent GJ. Idiopathic pulmonary fibrosis: multiple causes and multiple mechanisms? Eur Respir J. 2007; 30: 835-9.

3. Raghu G, Collard HR, Egan JJ, Martinez FJ, Behr J, Brown KK, et al. An Official ATS/ERSARS/ALAT Statement: Idiopathic Pulmonary Fibrosis: Evidence-based Guidelines for Diagnosis and Management. Am J Resp Crit Care. 2011; 183: 788-824.

4. Raghu G, Remy-Jardin M, Myers JL, Richeldi L, Ryerson CJ, Lederer DJ, et al. Diagnosis of Idiopathic Pulmonary Fibrosis An Official ATS/ERS/JRS/ALAT Clinical Practice Guideline. Am J Resp Crit Care. 2018; 198: E44-E68.

5. Aussedat PH, Chebib N, Ahmad K, Glerant JC, Drevet G, Grima R, et al. Impact of Lung Biopsy on Lung Function in Idiopathic Pulmonary Fibrosis. Respiration. 2021; 99: 1101-8. 
6. Hutchinson JP, Fogarty AW, McKeever TM, Hubbard RB. In-Hospital Mortality after Surgical Lung Biopsy for Interstitial Lung Disease in the United States. Am J Resp Crit Care. 2016; 193: 1161-7.

7. Ley B, Ryerson CJ, Vittinghoff E, Ryu JH, Tomassetti S, Lee JS, et al. A Multidimensional Index and Staging System for Idiopathic Pulmonary Fibrosis. Ann Intern Med. 2012; 156: 684-U58.

8. Raghu G, Rochwerg B, Zhang Y, Garcia CAC, Azuma A, Behr J, et al. An Official ATS/ERS/JRS/ALAT Clinical Practice Guideline: Treatment of Idiopathic Pulmonary Fibrosis An Update of the 2011 Clinical Practice Guideline. Am J Resp Crit Care. 2015; 192: E3-E19.

9. Noble PW, Albera C, Bradford WZ, Costabel U, Glassberg MK, Kardatzke D, et al. Pirfenidone in patients with idiopathic pulmonary fibrosis (CAPACITY): two randomised trials. Lancet. 2011; 377: $1760-9$.

10. Richeldi L, du Bois RM, Raghu G, Azuma A, Brown KK, Costabel U, et al. Efficacy and Safety of Nintedanib in Idiopathic Pulmonary Fibrosis. New Engl J Med. 2014; 370: 2071-82.

11. Management and prevention of Chronic Obstructive Pulmonary Disease, Global Initiative for Chronic Obstructive Lung Disease (GOLD). 2017.

12. Benton HP, Ivanisevic J, Mahieu NG, Kurczy ME, Johnson CH, Franco L, et al. Autonomous Metabolomics for Rapid Metabolite Identification in Global Profiling. Anal Chem. 2015; 87: 884-91.

13. Kanehisa M, Goto S, Sato Y, Furumichi M, Tanabe M. KEGG for integration and interpretation of largescale molecular data sets. Nucleic Acids Res. 2012; 40: D109-D14.

14. Li QP, Sun J, Mohammadtursun N, Wu JF, Dong JC, Li LL. Curcumin inhibits cigarette smoke-induced inflammation via modulating the PPAR gamma-NF-kappa B signaling pathway. Food Funct. 2019; 10: 7983-94.

15. Staiano RI, Loffredo S, Borriello F, lannotti FA, Piscitelli F, Orlando P, et al. Human lung-resident macrophages express $\mathrm{CB} 1$ and $\mathrm{CB} 2$ receptors whose activation inhibits the release of angiogenic and lymphangiogenic factors. J Leukocyte Biol. 2016; 99: 531-40.

16. Cinar R, Gochuico BR, lyer MR, Jourdan T, Yokoyama T, Park JK, et al. Cannabinoid CB1 receptor overactivity contributes to the pathogenesis of idiopathic pulmonary fibrosis. Jci Insight. 2017; 2.

17. Maher TM. Beyond the diagnosis of idiopathic pulmonary fibrosis; the growing role of systems biology and stratified medicine. Curr Opin Pulm Med. 2013; 19: 460-5.

18. Rosas IO, Richards TJ, Konishi K, Zhang Y, Gibson K, Lokshin AE, et al. MMP1 and MMP7 as potential peripheral blood biomarkers in idiopathic pulmonary fibrosis. Plos Med. 2008; 5: 623-33.

19. Herazo-Maya JD, Sun JH, Molyneaux PL, Li Q, Villalba JA, Tzouvelekis A, et al. Validation of a 52gene risk profile for outcome prediction in patients with idiopathic pulmonary fibrosis: an international, multicentre, cohort study. Lancet Resp Med. 2017; 5: 857-68.

20. Kang YP, Lee SB, Lee JM, Kim HM, Hong JY, Lee WJ, et al. Metabolic Profiling Regarding Pathogenesis of Idiopathic Pulmonary Fibrosis. J Proteome Res. 2016; 15: 1717-24.

21. Vaidya B, Patel R, Muth A, Gupta V. Exploitation of Novel Molecular Targets to Treat Idiopathic Pulmonary Fibrosis: A Drug Discovery Perspective. Curr Med Chem. 2017; 24: 2439-58. 
22. Patsenker E, Stoll M, Millonig G, Agaimy A, Wissniowski T, Schneider V, et al. Cannabinoid Receptor Type I Modulates Alcohol-Induced Liver Fibrosis. Mol Med. 2011; 17: 1285-94.

23. Lin CL, Hsu YC, Lee PH, Lei CC, Wang JY, Huang YT, et al. Cannabinoid receptor 1 disturbance of PPAR gamma 2 augments hyperglycemia induction of mesangial inflammation and fibrosis in renal glomeruli. J Mol Med. 2014; 92: 779-92.

24. Slavic S, Lauer D, Sommerfeld M, Kemnitz UR, Grzesiak A, Trappiel M, et al. Cannabinoid receptor 1 inhibition improves cardiac function and remodelling after myocardial infarction and in experimental metabolic syndrome. J Mol Med. 2013; 91: 811-23.

25. Cinar R, lyer MR, Liu ZY, Cao ZX, Jourdan T, Erdelyi K, et al. Hybrid inhibitor of peripheral cannabinoid1 receptors and inducible nitric oxide synthase mitigates liver fibrosis. Jci Insight. 2016; 1.

26. Rice W, Shannon JM, Burton F, Fiedeldey D. Expression of a brain-type cannabinoid receptor (CB1) in alveolar Type II cells in the lung: Regulation by hydrocortisone. Eur J Pharmacol. 1997; 327: 227-32.

\section{Figures}


A

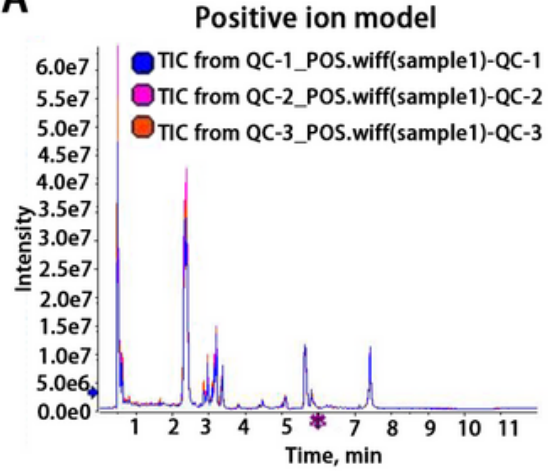

B

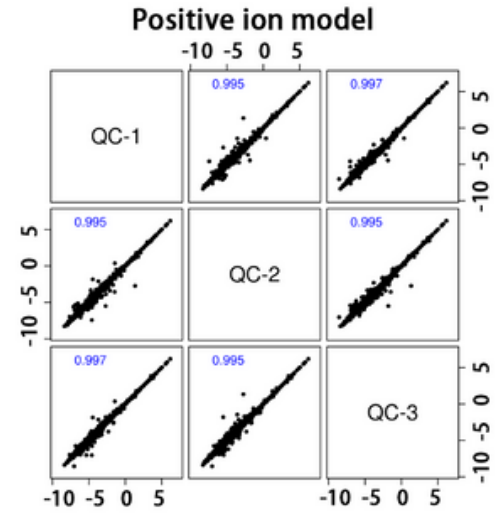

C

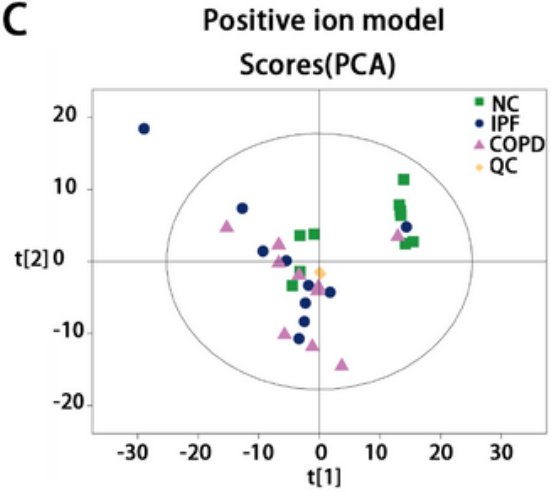

D

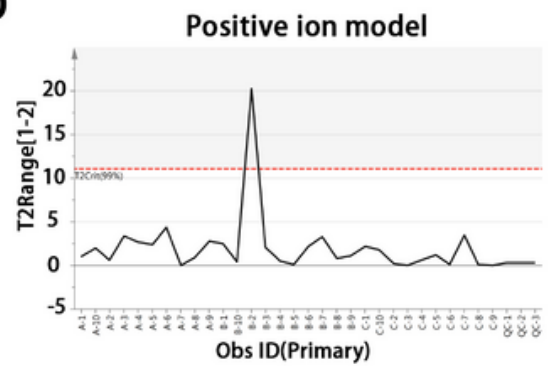

Negtive ion mode

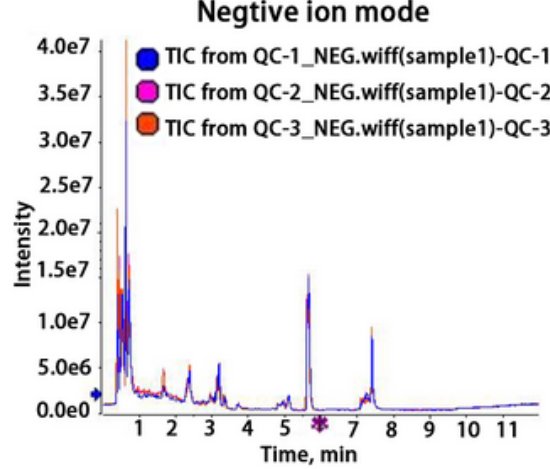

Negtive ion mode

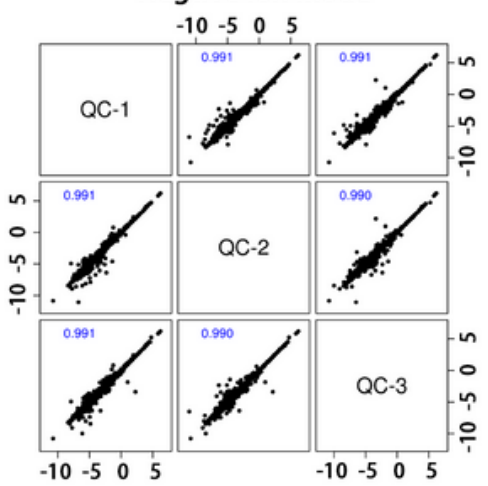

Negatvie ion model Scores(PCA)

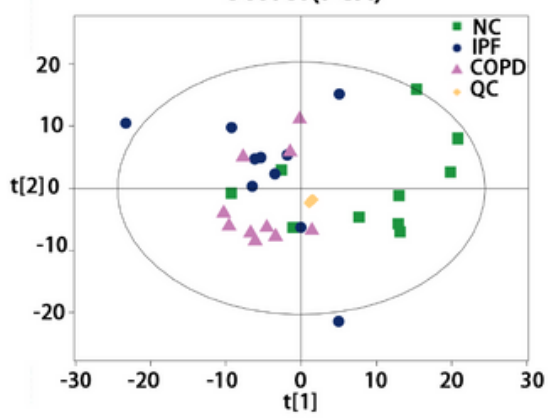

Negatvie ion model

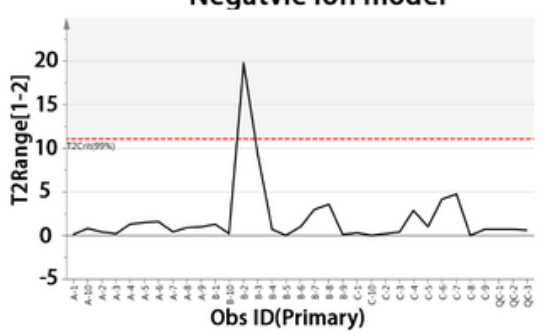

\section{Figure 1}

Optimazation of the analytical method and quality evaluation of LC-MS/MS analysis. (A). Total ion chromatogram (TIC) of quality control (QC). (B). Scatter plots of quality control. (C). Principal component analysis (PCA). (D). Hotelling's T2 chart of QC samples. 


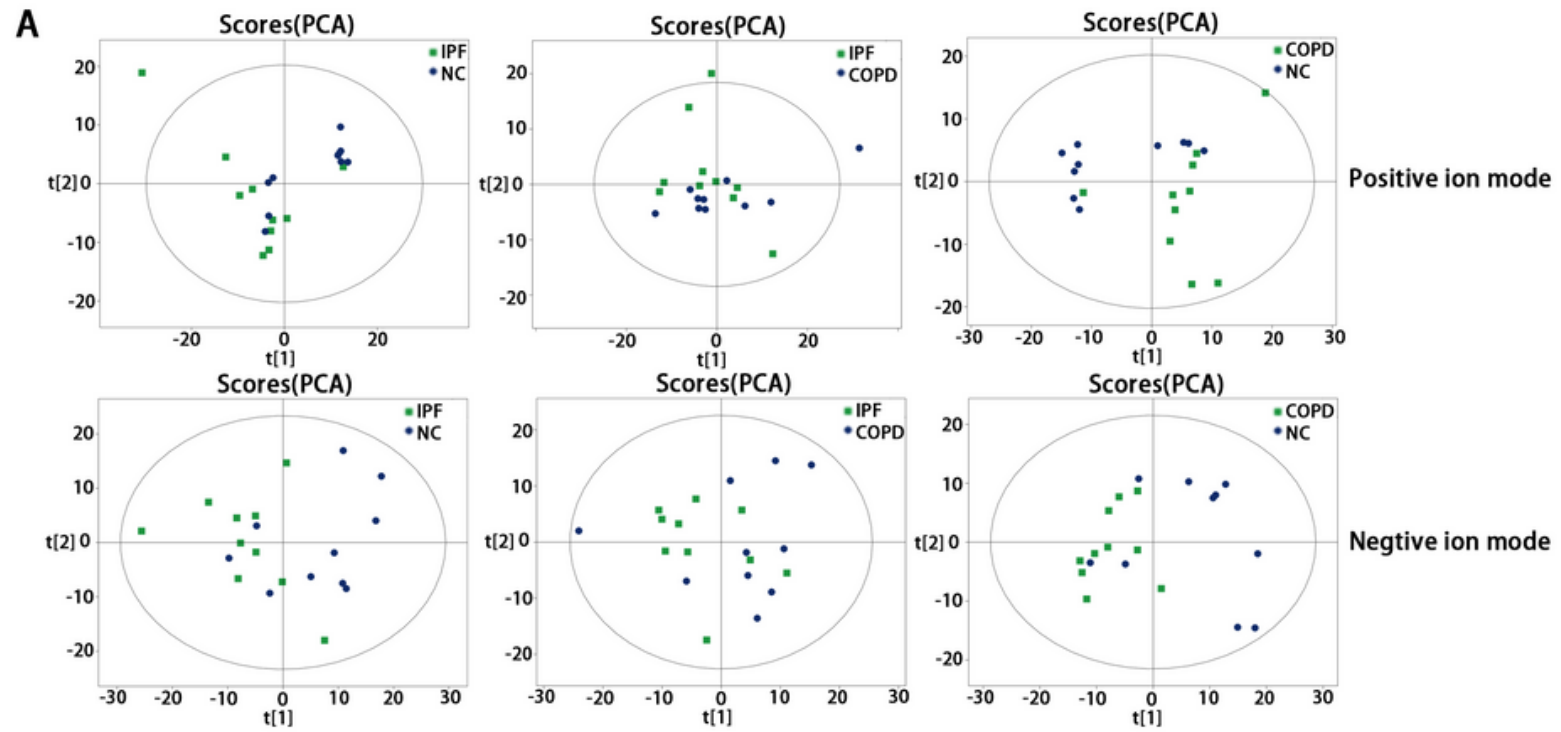

B
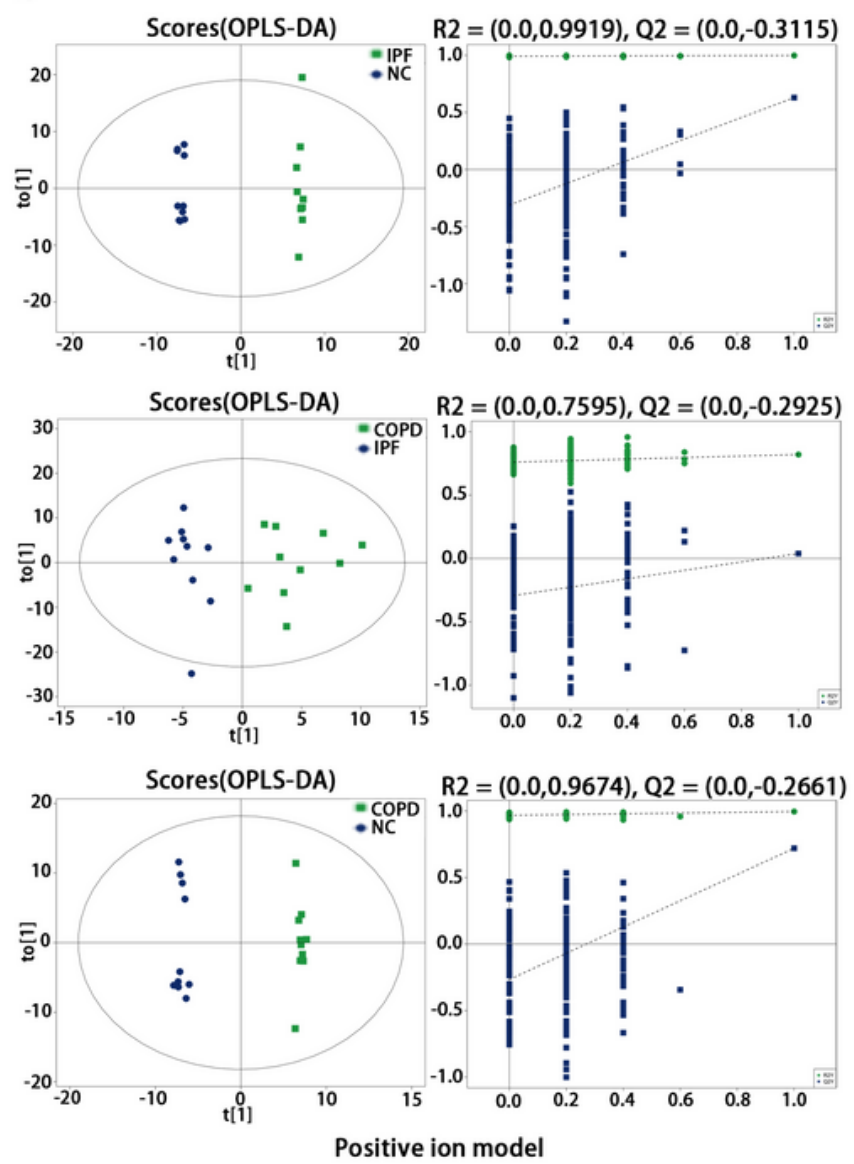

C
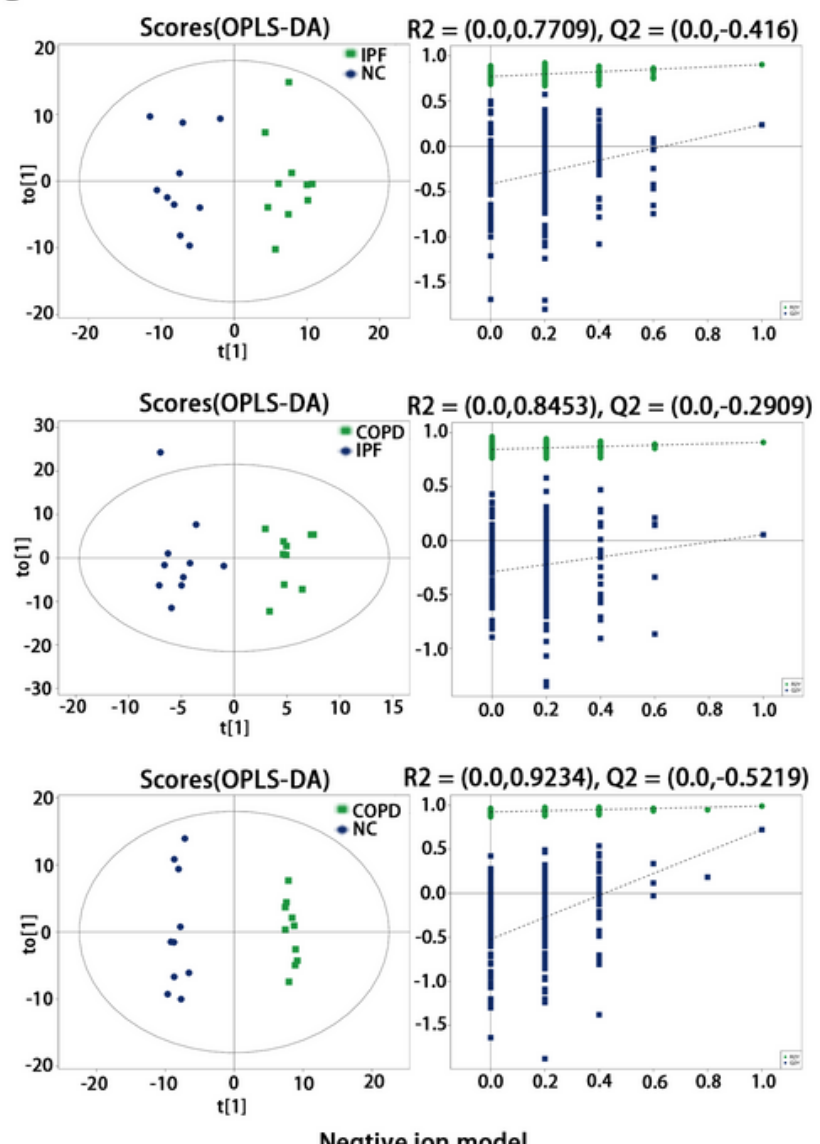

Negtive ion model

Figure 2

Multivariate analysis charts of NC, IPF and COPD samples. (A). PCA shot chart. (B). OPLS-DA score plots for NC, IPF and COPD samples in positive ion mode. OPLS-DA analysis results (panel left); OPLS-DA replacement inspection results (panel right). (C). OPLS-DA score plots in negative ion mode. 
A
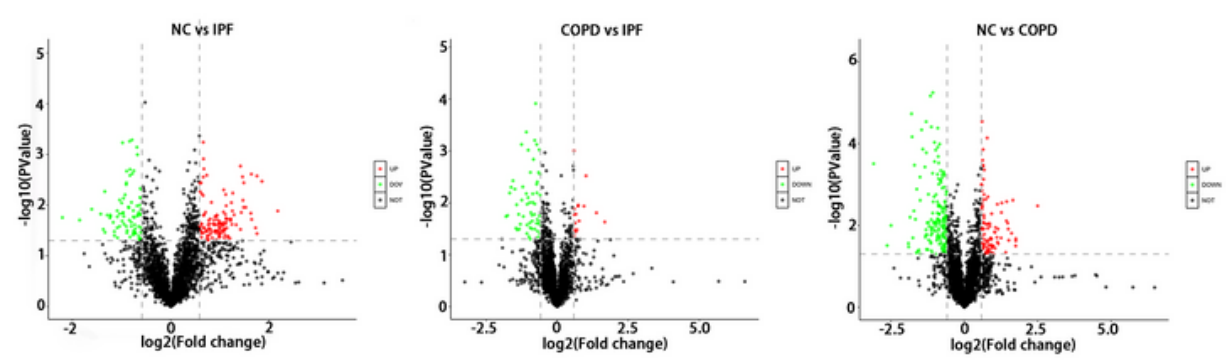

B

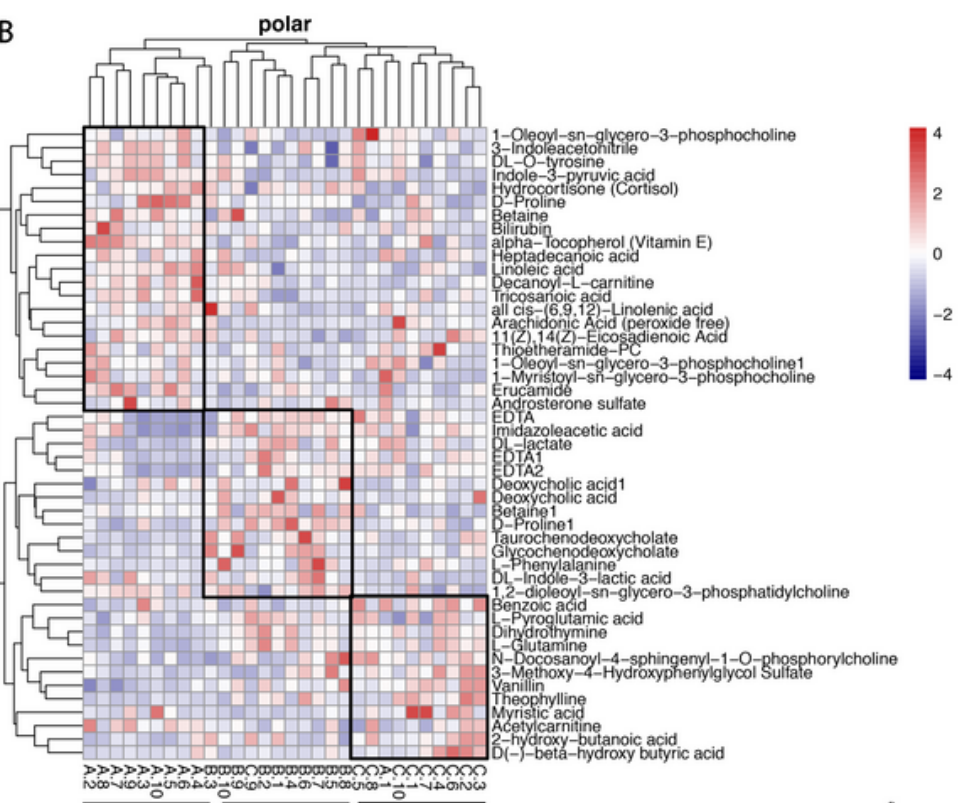

C

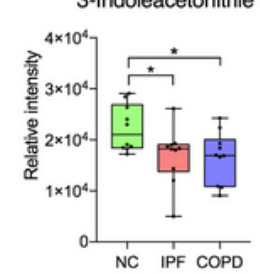

Glycochenodeoxycholate
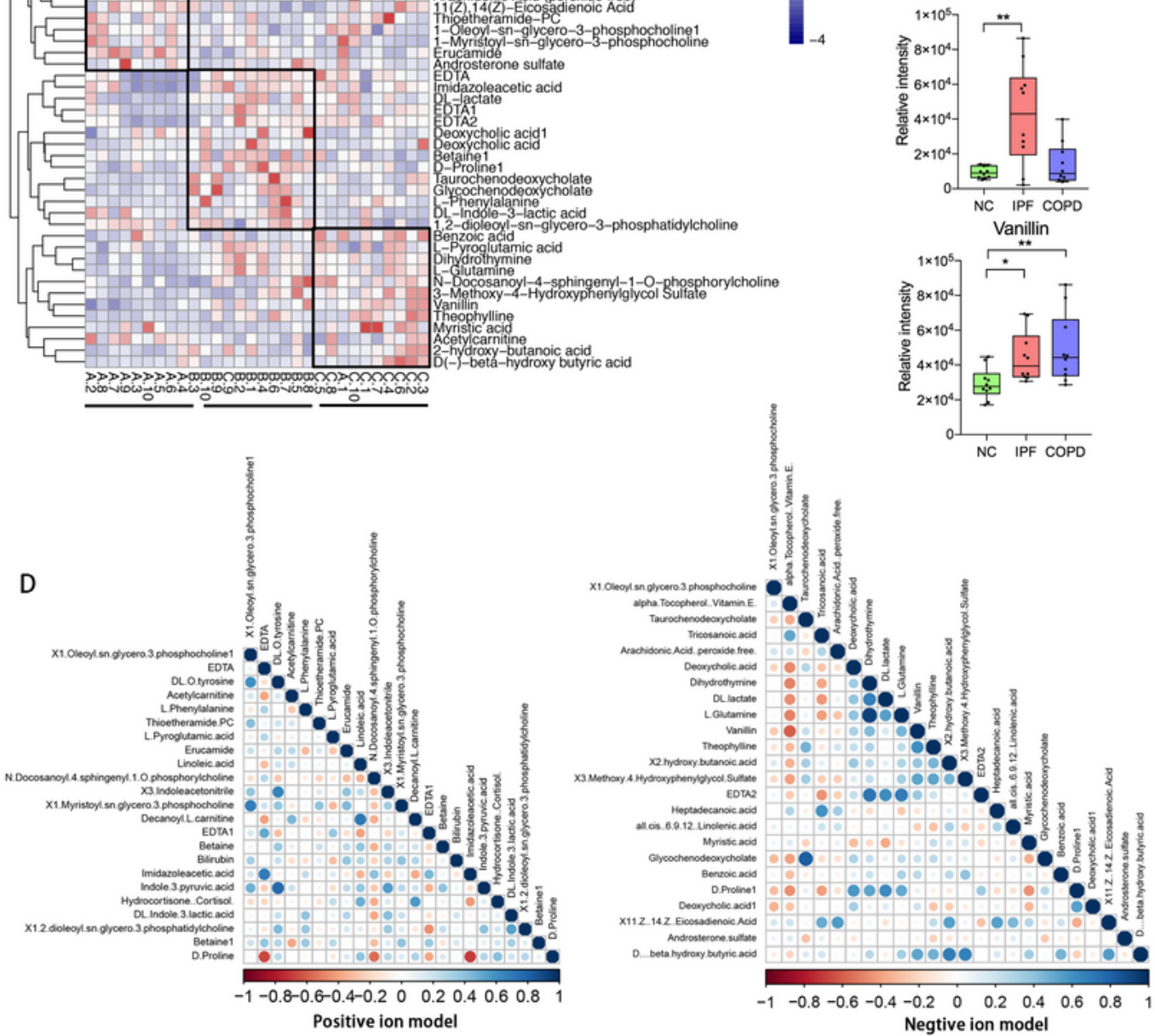

Figure 3

Summary of differential metabolites among NC, IPF and COPD. (A). Volcano Plot for screening of significantly changed metabolites $(F C>1.5$ and $P$ value $<0.05)$. (B). Heatmap visualization analysis of significantly changed metabolites. (C). Box plots of representative metabolite changes among NC, IPF and COPD groups. * $p<0.05,{ }^{* \star} p<0.01$. (D). Correlation analysis of the 24 pre-selected discriminating 
metabolites after comparison of IPF samples to NC and COPD samples in positive ion model (panel left) and in negative ion model (panel right).

\section{A}
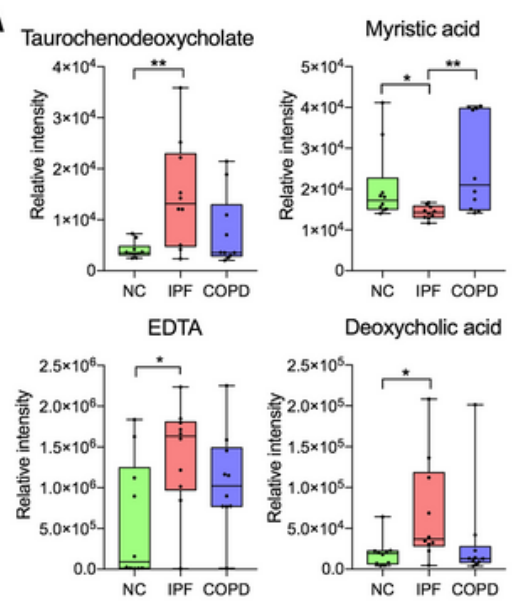

B

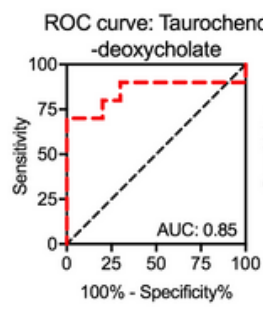

ROC curve: EDTA

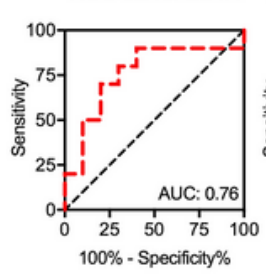

C

ROC curve: Taurocheno

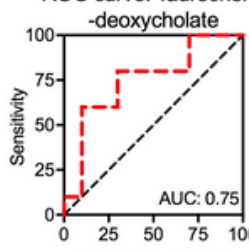

$100 \%$ - Specificity $\%$

ROC curve: EDTA
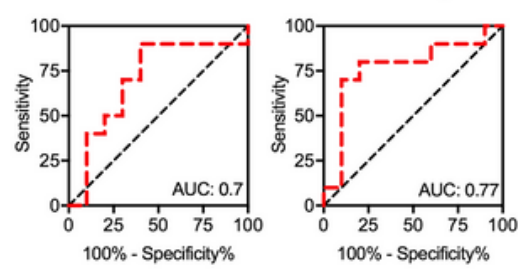

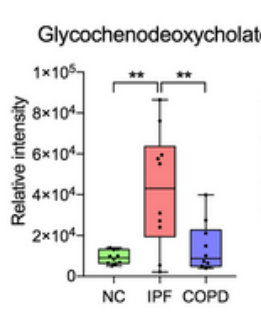

D-Proline

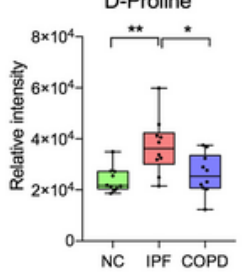

NC vs IPF

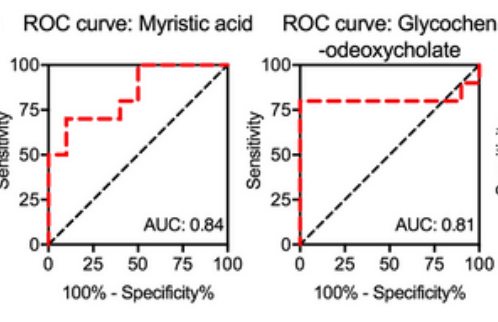

ROC curve: Erucamide

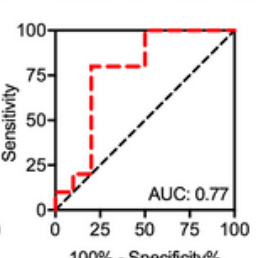

$100 \%$ - Specificity $\%$
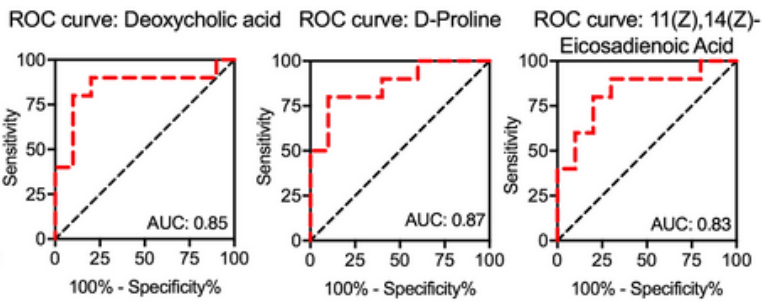

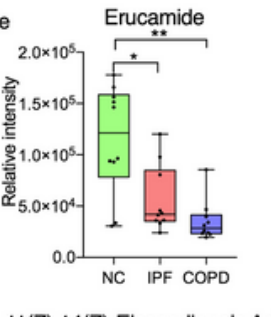

11(Z),14(Z)-Eicosadienoic Acid

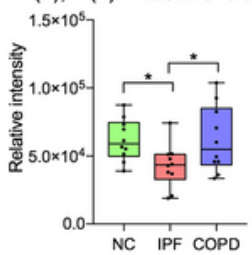

IPF vs COPD

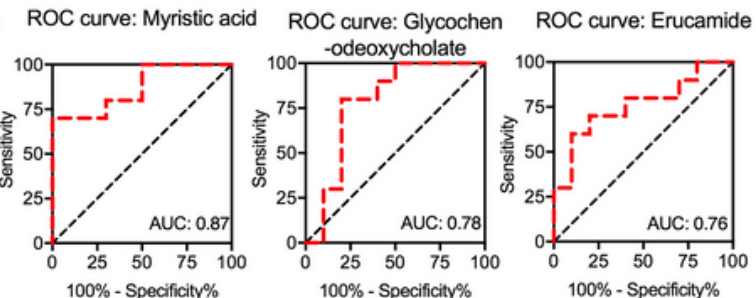

ROC curve: Deoxycholic acid ROC curve: D-Proline
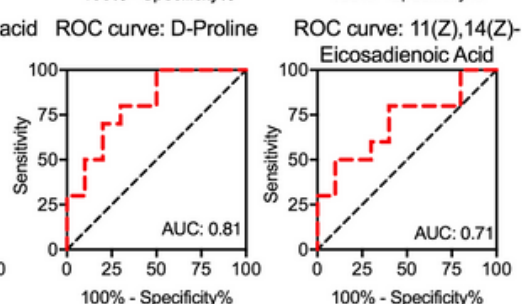

\section{Figure 4}

ROC curves of differential metabolites among IPF, COPD and control subjects with AUC $\geq 0.7$. (A). ROC curves analysis of 8 metabolites for discriminating IPF objects from controls. (B). ROC curves analysis of 8 metabolites for discriminating IPF objects from COPD. 

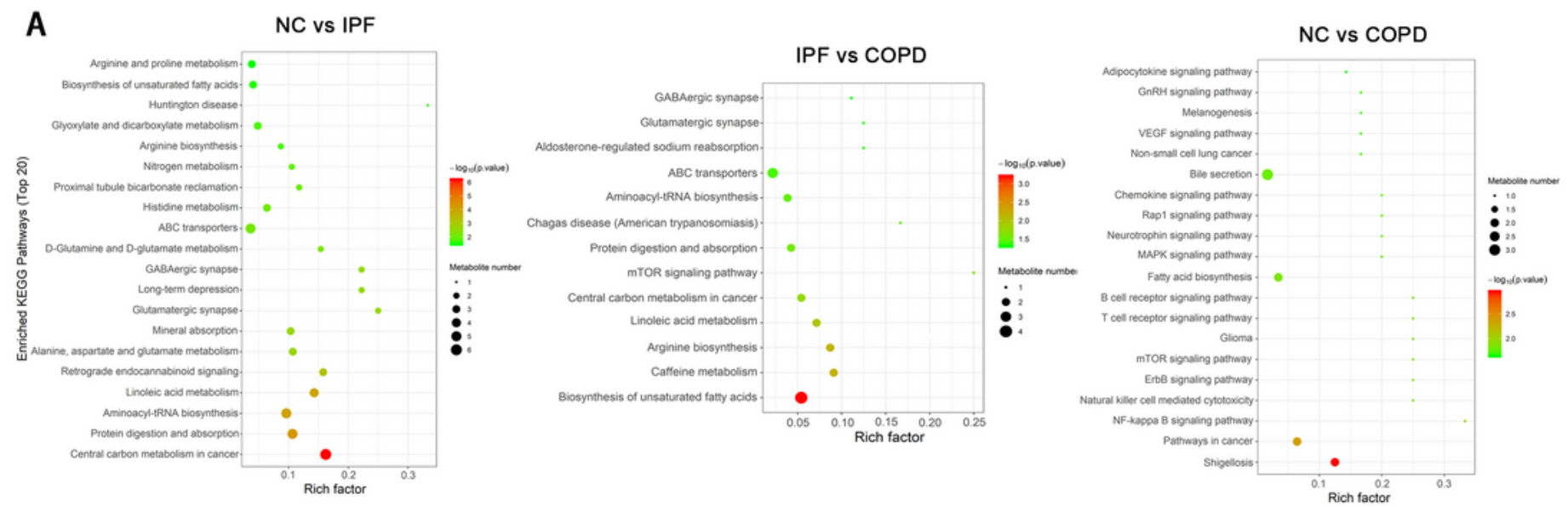

B RETROGRADE ENDOCANNABINOID SIGNALING
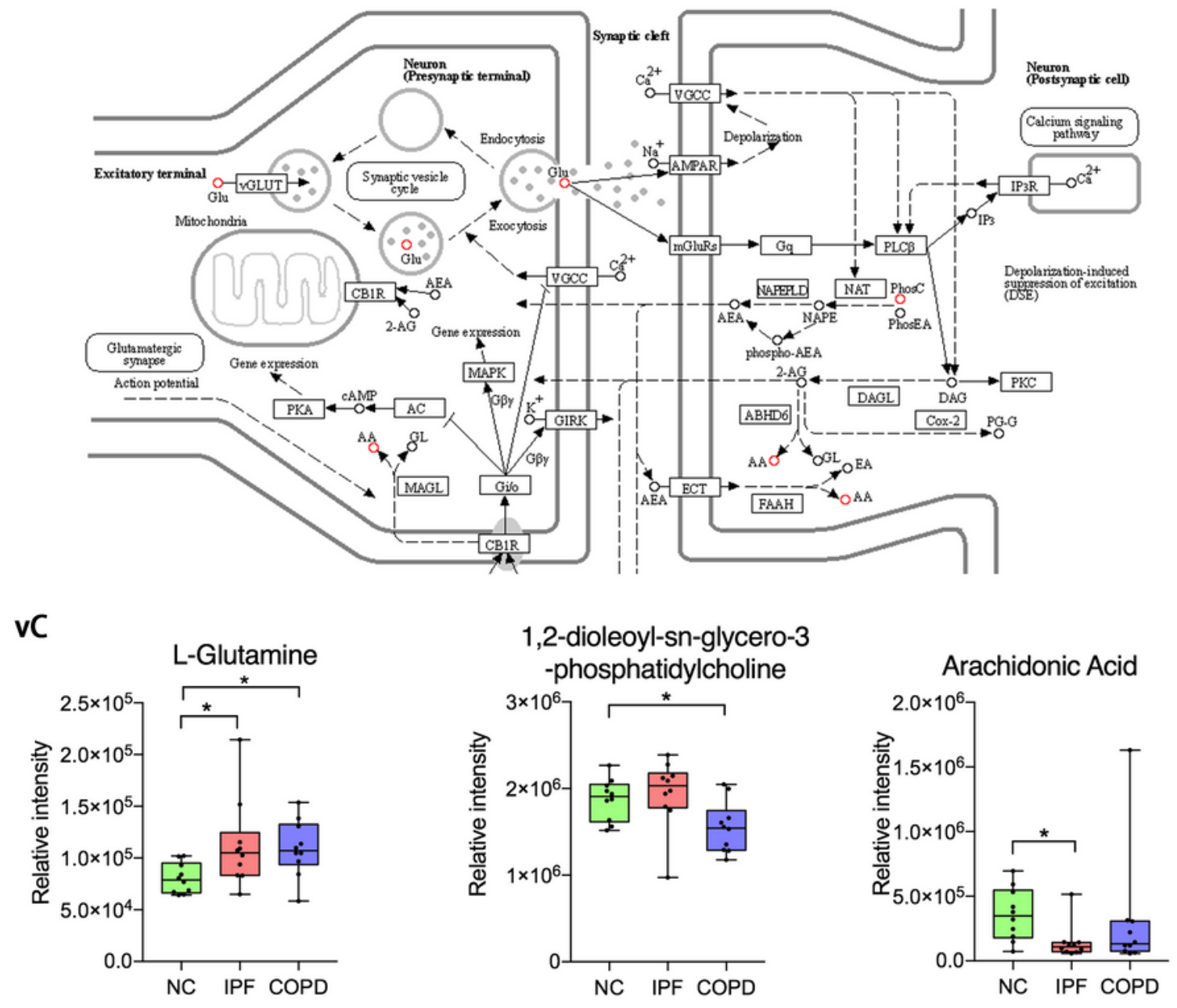

\section{Figure 5}

KEGG pathway analysis and bubble chart shows enrichment of differential metabolites in signaling pathways. (A). Bubble chart of KEGG pathway enrichment analysis results. (B). The key target of the Endogenous Cannabinoids/Cannabinoid Receptor 2 pathway in IPF. Metabolite with red color in this reference map represents an changed level of this metabolite in IPF subjects. (C). Box plots of deregulated metabolites found in Endogenous Cannabinoids pathway. 

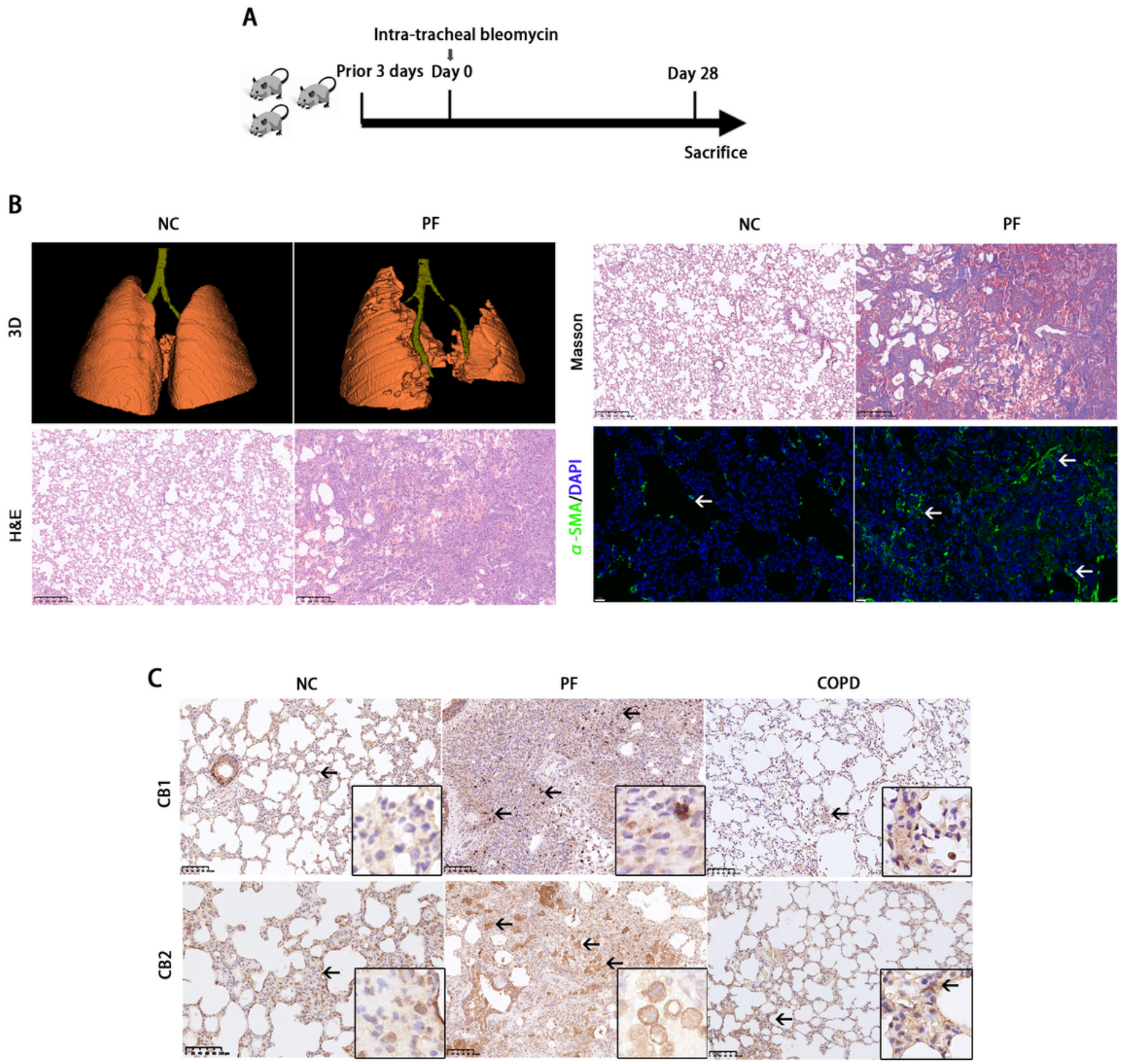

\section{Figure 6}

Representative pirctures of $\mathrm{IHC}$ staining for rat $\mathrm{PF}$ model and cannabinoid receptor $1 / 2$ protein in normal control and PF lung tissues. (A). Diagram of experimental protocol for BLM-induced pulmonary fibrosis. (B). Representative images of axial slices of reconstructed micro-CT images obtained at last day post treatment. Histopathological and fibrotic changes in the lungs of rats post BLM injury. Images of Masson's trichrome, hematoxylin and eosin stained lung sections (scale bar: $250 \mu \mathrm{m}$ ). Representative

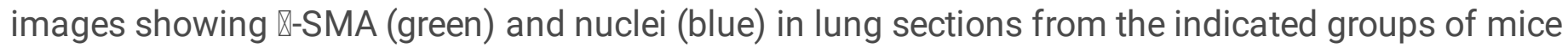
(scale bar: $50 \mu \mathrm{m}$ ). (C). Immunohistochemical staining of lung tissue with anti-CB1 and anti-CB2 antibody (scale bar: $250 \mu \mathrm{m}$ ). The same field of view as in panel presented at $\times 100$ magnification. 